\title{
Calibration of X-Ray Measurement of Strain
}

\author{
By John A. Bennett and Herbert C. Vacher
}

\begin{abstract}
In order to increase the precision of strain determination by X-ray methods, 12 measurements of ring radius were made on each of two patterns for the customary incidence angles of $90^{\circ}$ and $45^{\circ}$. For a specimen to which uniaxial stress was applied, a method was developed for handling the data to give one value representative of these 24 readings. When these values (obtained on a flat steel specimen loaded in bending) were compared with mechanical strain measurements, it was found that the X-ray measurements were proportional to the maximum principal strain up to the beginning of plastic deformation, and the precision was such that a change corresponding to $1,000 \mathrm{lb} /$ in..$^{2}$ should be detectable.
\end{abstract}

\section{Introduction}

In an investigation at the National Bureau of Standards it was necessary to determine strain by means of X-rays. As the accuracy required was greater than that ordinarily obtained with this method, experiments were made to develop a technique for making the measurements with sufficient accuracy for the purpose intended. The method developed and some results obtained with it are described in this paper.

Deformation of metals can be of two types, elastic and plastic. Elastic deformation results in a change in the interatomic spacing of the crystals, whereas plastic deformation does not. It is possible, by means of X-ray diffraction, to measure the lattice spacing and thus determine elastic strain independently of plastic strain. Because of this and other advantages over mechanical extensometers, which measure over all changes in dimensions, X-ray diffraction has been widely used for the determination of strain and stress in metals. During the past 20 years the literature on the subject has become extensive ${ }^{12}$. A very good general treatment is given by Barrett. ${ }^{3}$

The $\mathrm{X}$-ray strain measurements are made on relatively few crystals oriented in particular directions, and it is possible that the deformation of

\footnotetext{
${ }^{1}$ Herbert R. Isenburger, Welding Research Supplement, Welding J. 23, 571-s (Nov. 1944).

${ }^{2}$ George Sachs, C. S. Smith, J. D. Lubahn, G. E. Davis, and L. J. Ebert, Welding Research Supplement, Welding J. 25, 400-s (July 1946).

${ }^{3}$ C. S. Barrett, Structure of metals, chap. 14, p. 267 (McGraw-Hill Book Co., Inc., New York, N. Y., 1943).
}

these crystals is not representative of the behavior of the polycrystalline material as a whole. Also the strain cannot be measured in the direction of the applied stress, so the principal strains must be calculated by means of elastic theory. The uncertainty introduced by these two factors must be overcome by experiment to ensure the accuracy of the X-ray determination of strain. In order to do this and to evaluate the precision of the method, calibrating tests were made on a specimen to which uniaxial stress was applied.

\section{X-Ray Diffraction Method of Measuring Strain}

\section{Precision of a Single Measurement}

The mathematical relationships involved in the measurement of strain for the case of uniaxial stress will be reviewed before describing the method. In the back-reflection technique of X-ray diffraction, the collimated beam is passed through a photographic film placed at right angles to it. The beam strikes the specimen and is diffracted by those crystals which are so oriented as to satisfy the Bragg equation

$$
n \lambda=2 d \sin \theta,
$$

where $n$ is the order, $\lambda$ the wavelength of the $\mathrm{X}$-rays, $d$ the interplanar spacing, and $\theta$ the diffraction angle. If monochromatic X-rays are used, the incident beam is diffracted into a series of cones having a common axis coincident with the incident beam; if any one of these intersects 
the photographic film, a circle will be recorded. When $d$ is changed by the application of stress to the material, $\sin \theta$ changes correspondingly, as the product $n \lambda$ is constant.

In considering the relationships involved in the determination of strain by X-ray diffraction, the assumptions are made that the material is isotropic and homogeneous, and that the applied stress is uniaxial. The coordinate system is set up with the $X$ and $Y$ axes in the surface of the specimen and the $Z$ axis normal to the surface: The stress is applied parallel to the $X$ axis. Figure 1 shows the relationship of various angles

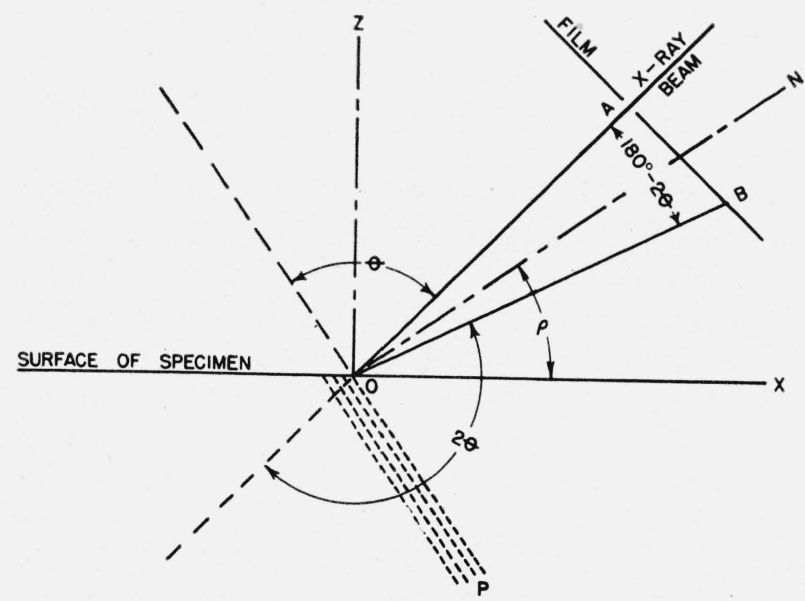

FIGURE 1. Relationship of various angles in a plane normal to the surface of the specimen and parallel to the applied stress.

Reflecting planes are shown at $P$, and the diffracted beam intersects the film at $B$.

in the $X Z$ plane when the incident $\mathrm{X}$-ray beam makes an oblique angle with the surface of the specimen. The diffracted beam is shown intersecting the photographic film at $B$, one set of reflecting planes is shown at $P$, and $N$ is the normal to these planes. The intersection of the diffracted cone with the $X Z$ plane on the other side of the incident beam has been omitted to avoid confusing the diagram. In order to determine $\theta$, the distances $O A$ and $A B$ are measured.

Definitions of other symbols used are as follows: $S=$ Specimen-to-film distance $(O A$ on fig. 1).

$R=$ Radius of the diffraction ring on the photographic film ( $A B$ on fig. 1).

$\rho=$ Angle between the $X$ axis and the normal to the reflecting planes. $\beta=$ Longitude angle about the incident $\mathrm{X}$-ray beam. The zero position is in the $X Z$ plane on the side of the incident beam nearest the positive $X$ direction.

$\epsilon=$ Strain in a direction making an angle $\rho$ with the $X$ axis.

$\epsilon_{X}, \epsilon_{Y}, \epsilon_{Z}=$ Strain in the $X, Y$, and $Z$ directions, respectively.

$\nu=$ Poisson's ratio; assumed to equal 0.3 .

Measurements of strain by the X-ray method are not made in the direction of the applied stress, therefore, it is necessary to know how the strain varies with direction. This is shown (for uniaxial stress parallel to the $X$-axis) in figure 2 , which is a stereographic projection, the plane of projection being in the surface of the specimen ( $X Y$ plane). The latitude lines connect points of equal strain ratio. Strain ratio, defined as the ratio of the strain in a given direction to that in the direction of the applied stress is designated as $\boldsymbol{\epsilon} / \boldsymbol{\epsilon}_{X}$.

The formula used in computing the strain ratios

$$
\epsilon / \epsilon_{X}=\cos ^{2} \rho-0.3 \sin ^{2} \rho,
$$

was derived from the approximate equation for the ellipsoid of strain, the complete derivation being shown in section VI, 1.

Obviously it is desirable to make strain measurements at an angle such that the strain ratio will be as large as possible, but mechanical considerations limit the incident angle to a maximum of about $45^{\circ}$ from the normal to the surface of the specimen. On figure 2 are shown the reflection circles (cones formed by the normals to the reflecting planes) for two positions of the incident beam; normal to the surface and at $45^{\circ}$ to the surface in the $X Z$ plane. The circles were calculated for cobalt $K \alpha$ radiation reflected from the 310 planes of iron, the diffracting angle, $\theta$, being $80 \frac{1}{2}{ }^{\circ}$. Position on the reflection circle is defined by $\beta$. The point of maximum strain ratio coincides with the position $\beta=0$ when the beam is incident at $45^{\circ}$, and for this condition the strain ratio is 0.562 , computed from eq. 2 .

In order to calculate the change in $\tan 2 \theta$ (which is the quantity measured experimentally), that would be expected for a given strain, eq 1 is differentiated (see section VI, 2 for complete derivation), and it is found that for infinitesimal strains, 


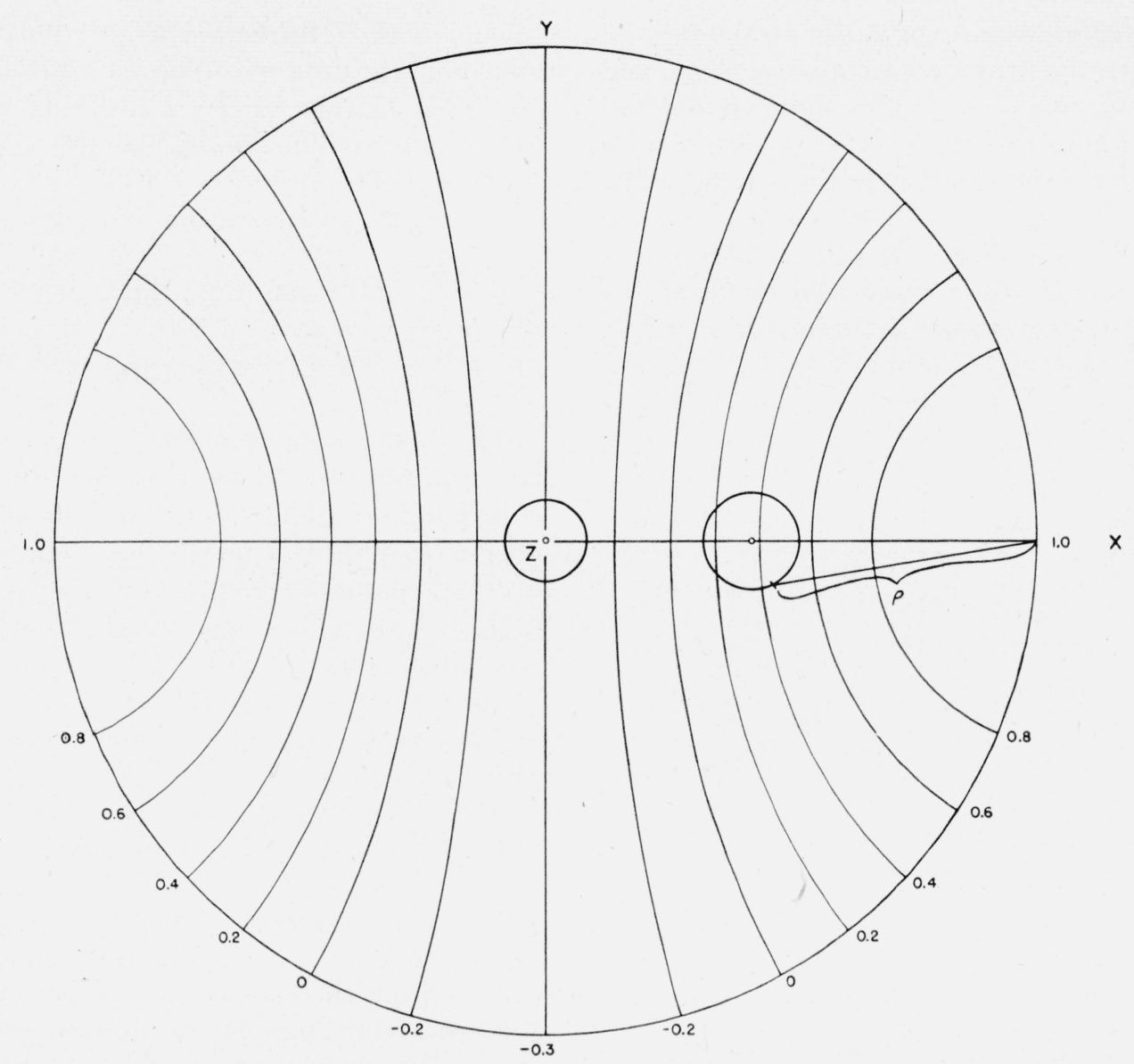

FIGURE 2. Stereographic projection (plane of projection is the surface of the specimen) showing the variation of strain ratio with direction (latitude circles), for uniaxial loading in the $X$ direction.

The small circles are the reflection circles for X-ray beams incident at $90^{\circ}$ and $45^{\circ}$. Cobalt $K \alpha$ radiation reflected from the 310 planes of iron.

$$
\epsilon=-\left[\frac{\left(1-2 \sin ^{2} \theta\right)^{2}}{2 \tan \theta}\right] d \tan 2 \theta
$$

The term in brackets is nearly constant ${ }^{4}$ over the range of values of $\theta$ involved in this work, so tan $2 \theta$ can be considered to vary linearly with strain. Assuming that the maximum strain which annealed medium carbon steel can withstand without plastic deformation is about 0.001 , the strain at the point of maximum available strain ratio would be 0.00056 . Substituting this value in eq 3 and assuming $\theta=80 \frac{1 / 2}{2}$, the change in $\tan 2 \theta$ is found to be 0.0075 . In work of this kind the specimen-to-film distance is usually about $60 \mathrm{~mm}$; at this distance the above change in $\tan 2 \theta$ would cause a change of $0.45 \mathrm{~mm}$ in the radius of the diffraction ring. As the radii of the sharpest

\footnotetext{
${ }^{4}$ The errors involved in certain of the arfroximations made in this paper
} are evaluated in section VI, 4. diffraction rings cannot be measured more closely than about $0.05 \mathrm{~mm}$, the sensitivity of a single measurement is greater than 10 percent of the maximum strain to be determined. This con. sideration made it obvious that the number of measurements used to determine a specific condition of strain would have to be farily large if the required precision were to be obtained.

\section{Strain Determination From Multiple Measure- ments of Ring Radius}

It will be noted on figure 2 that the reflection circles (particularly when the incident angle is $45^{\circ}$ ) intersect a wide range of values of strain ratio. This means that the radius of the diffraction ring from a stressed specimen will not be constant, as was shown by Stäblein. ${ }^{5}$ Figure 2

${ }^{5}$ Von F. Stäblein, Tech. Mitt. Krupp 2, 29 (appendix, 1939). 
shows that for all values of $\beta$ the strain ratio is negative when the beam is normally incident, and positive for $45^{\circ}$ incidence. This is shown in detail in figure 3 , where the strain ratio is plotted as a function of position on the reflection circle. The values used in plotting these curves can be obtained either by determining $\rho$ for various values of $\beta$ on a stereographic projection or from the geometrical considerations shown in section VI, 3 .

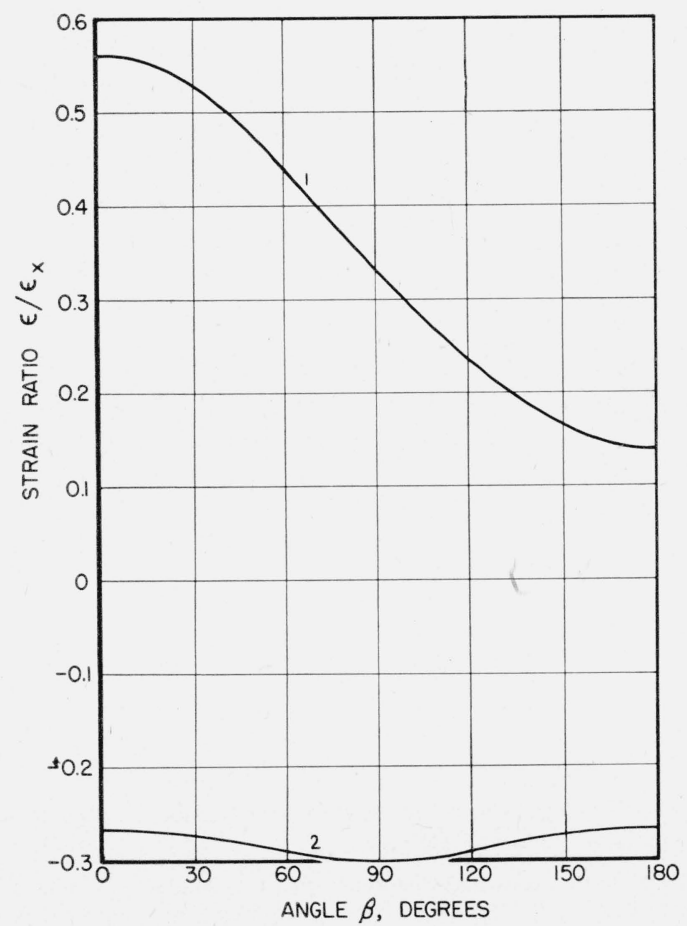

FIGURE 3. Theoretical variation of strain ratio with position on the diffraction ring, for uniaxial stress.

$1,45^{\circ}$ incidence; $2,90^{\circ}$ incidence.

It should be pointed out that the curves of figure 3 are strictly true only for infinitesimal values of strain, since $\theta$, and therefore $\rho$, change slightly with strain. As shown in section VI, 4 the effect of the maximum anticipated strain on the position of the curves of figure 3 is not significant.

As was shown above, $\tan 2 \theta$ can be considered to be a linear function of strain; therefore, if the measured values of $\tan 2 \theta$ for various positions on the ring are plotted against the corresponding values of the strain ratio, the points would be expected to lie on a straight line. The intercept of this line at $\beta=0$, for example, would provide a value representative of all the measurements taken on that diffraction ring. This method of analyzing the data was followed in the experiments described in this report in order to increase the precision over that obtainable with a single measurement. The results will be discussed after the experimental procedures have been described.

\section{Procedure}

\section{Apparatus}

The X-ray apparatus used in this work was a General Electric XRD unit, the tube having a cobalt target. The voltage was $45-47 \mathrm{KVP}$ with a tube current of $7 \mathrm{ma}$. The collimator consisted of two 1-mm-diameter pinholes spaced $75 \mathrm{~mm}$ apart. Under these conditions an exposure of 1 hour on Eastman No-Screen film was satisfactory. Single-coated film was not found to be necessary in this work, as only the change in radius was important.

A flat specimen was used, the stress being applied by bending. The specimen was made from a $5 / 8$-in. diameter rod of $\mathrm{X} 4130$ steel in the normalized condition. A flat central section, $1 / 16$ in. thick by $1 \frac{3}{3}$ in. long was machined equidistant from the ends of the rod, the thickness of the reduced section being uniform within 1 percent. The surface on which strain measurements were to be made was polished with progressively finer abrasive papers, finishing with 400 Aloxite; then the specimen was normalized in vacuum at $1,625^{\circ} \mathrm{F}$, and finally the surface was etched for 5 minutes in 1 percent nital.

Strain in the $X$-direction in the specimen was measured with two wire strain gages placed above and below the spot where the X-ray beam was incident. Baldwin-Southwark $\mathrm{A}-7$ gages, having an effective length of $1 / 4$ in., were read on a type $\mathrm{K}$ strain indicator. The average difference between the readings of the two gages was about $15 \times 10^{-6}$.

The specimen was loaded in a jig (a top view of which is shown in fig. 4), which applied a uniform bending moment to a 1-in. length of the reduced section. This was accomplished by loading the specimen as a simple beam, with the load distributed symmetrically on the two knife edges. This symmetry was maintained by supporting the knife edges on two pins, which allowed rotation about both a vertical and a horizontal axis. 


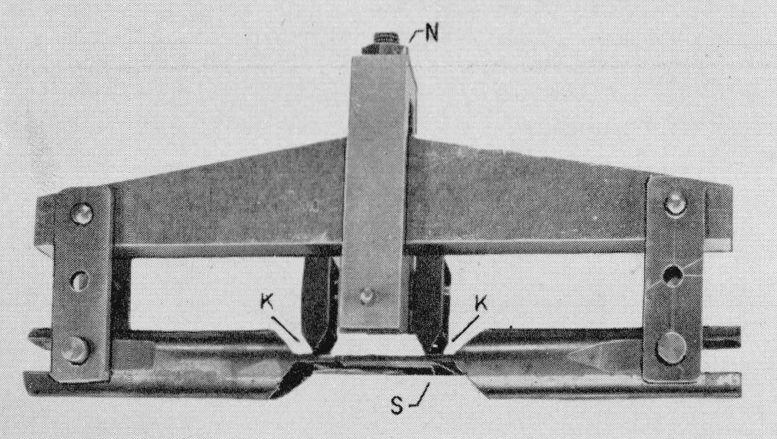

FiguRe 4. Jig for applying a bending moment to the flat specimen used in this investigation.

$S$, specimen; K, knife edges; $N$, loading nut.

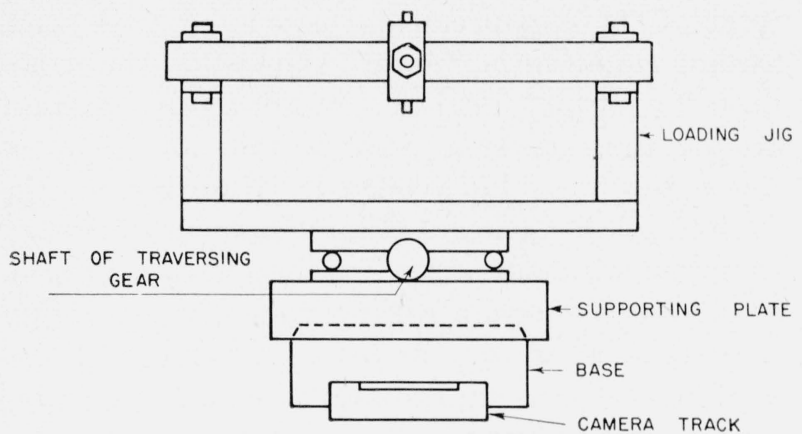

Figure 5. Sketch of complete mounting assembly for loading jig, looking toward the X-ray tube.

The loading jig was supported on three balls guided by steel $V$-blocks on the bottom plate of the jig and on the supporting plate below, as shown in the sketch of figure 5. The surface of the specimen was parallel to the $V$-blocks, so that the jig could be traversed while keeping the surface of the specimen accurately in the same plane. The supporting plate was mounted on a base in :such a way that the specimen could be set either normal to the incident beam or at an angle of $45^{\circ}$ to it when the base was in place on the track of the X-ray unit. During the exposure, the loading jig was translated horizontally about $3 \mathrm{~mm}$ by a clock mechanism operating through a flexible :shaft.

\section{Measurement of Film-to-Specimen Distance}

Two measurements are required for a determination of the diffraction angle, namely, the film-to-specimen distance, $S$, and the radius of the diffraction $\operatorname{ring}, R$, tan $2 \theta$ being $R / S$. Many investigators ${ }^{6}$ have, in effect, made the first

${ }_{6}$ See footnote 3 . measurement by using a calibrating substance in the form of a powder dusted on the specimen. The diffraction ring from this standard is compared with that from the specimen so that no direct measurement of the specimen-to-film distance is required. Because of the uncertainty of measurement of a diffraction ring, the more precise method of determining $S$ by micrometer measurement was adopted. These measurements were made on a separate camera track on which the complete specimen mount assembly was placed before making an exposure. A pointer controlled by a micrometer screw was mounted in a collimator mount on the camera track in such a way that the pointer was in the same position relative to the specimen as the $\mathrm{X}$-ray beam. The spacing between the collimator mount and the supporting plate of the specimen mount was set by means of a gage block placed on the camera track between them.

After the specimen mount assembly and the collimator mount had been clamped firmly against the gage block, the pointer was advanced until it contacted the specimen, the contact being indicated electrically. The micrometer reading was recorded and the complete specimen mount assembly was then transferred to the camera track on the X-ray apparatus. The same gage block was used to adjust the spacing between the supporting plate and the camera mount, following which the exposure was made. After a few calibrating exposures had been made with substances of known diffraction angle, the micrometer readings could be converted directly to values of $S$. This distance was approximately $60 \mathrm{~mm}$.

\section{Measurement of Ring Radius}

In order to provide a reference mark on the film from which to measure the radius of the diffraction ring, a calibrating ring was exposed on the film after the diffraction pattern. A circular mask having a diameter about $10 \mathrm{~mm}$ greater than the diffraction ring was held firmly against the light shield in such a way that the periphery of the mask was accurately concentric with the position of the collimated X-ray beam. A direct beam of X-rays, arranged to form a sharp shadow of the mask, produced a dark ring on the film, and this ring was used as a reference in all measurements of the diffraction ring. As the calibrating ring was exposed immediately after the diffraction 
pattern, any dimensional change in the film could be measured.

The diffuseness of the diffraction ring makes it difficult to measure the radii with the desired sensitivity of $0.05 \mathrm{~mm}$, the total width of the line being $1 \frac{1}{2}$ to $2 \mathrm{~mm}$, under the best circumstances. Preliminary measurements made for comparison on a recording microphotometer and with visual methods, indicated that the greater precision of the former justified the additional time which it required. The microphotometer used was of the Knorr-Albers type.

A film holder (fig. 6) was constructed which allowed the film to be rotated without being removed from the holder. The film was mounted between two circular glass plates which were held in a flanged ring by spring clamps. The ring was a close fit in a hole in a 4 - by 10 -in. brass plate and was beveled on the outer edge so that it could be held firmly against the plate by means of knurled clamping screws. The plate fitted into the plate holder of the microphotometer with the long axis of the plate horizontal. The calibrat-

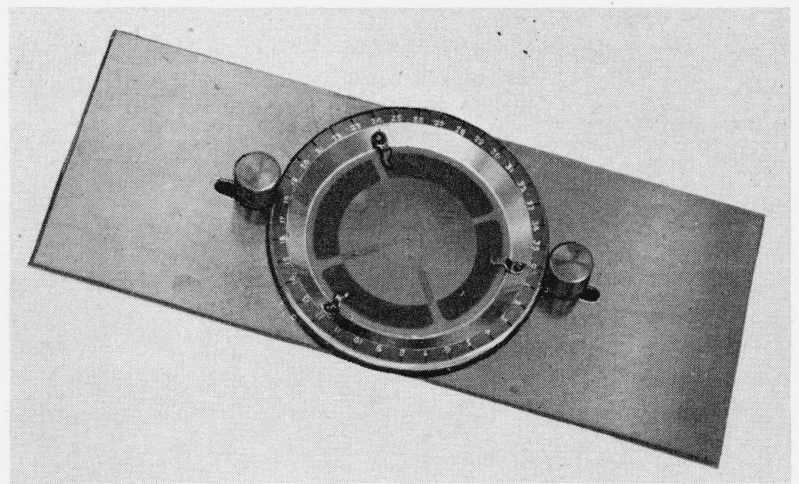

Figure 6. Film holder for mounting and rotating the diffraction pattern in the microphotometer.

ing ring on the film was made concentric with the ring of the film holder by means of $1 / 16$-in.diameter alinement holes in the center of the glass plates and in the film. Then the plate holder was adjusted so that the scanning light would pass through this common center. In this way the ring could be set quickly (using a graduated circle on the flange) for scanning any desired diameter of the diffraction ring. It was found by trial that the most satisfactory scanning speed was $10 \mathrm{~mm}$ a minute, which, with a chart speed of 2 in. a minute, gave a magnification of about $\times 5$ on the chart.
The diameter of the calibrating ring was measured at three positions on the film, and the distance from the calibrating ring to the diffraction ring was measured at 12 points $30^{\circ}$ apart. From these measurements the radii (corrected for film shrinkage) at 12 values of $\beta$ could be obtained, as the diameter of the calibrating ring was accurately known.

\section{Treatment of Data}

For each load applied to the specimen, two diffraction patterns were made, one with the $\mathrm{X}$ ray beam incident at an angle of $90^{\circ}$ and the other with $45^{\circ}$ incidence. As 12 measurments of ring radius were made on each pattern, there were a total of 24 measurements associated with each loading condition. Figure 3 shows that the strain ratio for normal incidence is sufficiently constant around the reflection circle so that all values of the radius may be averaged. However, in the case of $45^{\circ}$ incidence a considerable loss in sensitivity would result if the readings were averaged, as the average strain ratio is significantly smaller than that at $\beta=0$. Instead, the values of tan $2 \theta$ were plotted against strain ratio, and the intercept corresponding to $\beta=0$ was taken as the value representative of the group of readings. A typical set of data plotted in this way is shown in figure 7 , in which the coordinate lines are drawn at each value of $\beta$ where a measurement of radius was made. The strain in the $X$ direction $\left(\epsilon_{X}\right)$ in this case was 0.06 percent. The straight line representative of the points was determined by least squares analysis.

Both the slope and the intercept of curves plotted as in figure 7 would be expected to vary with strain, but the intercept was used as it was more sensitive to strain changes and could be compared more readily with the results from the patterns taken at normal incidence.

A systematic error is indicated by the fact that the points in figure 7 for the radii from 0 to $180^{\circ}$ are consistently higher than those from 180 to $360^{\circ}$. This discrepancy occurred in all patterns, regardless of angle of incidence, and is probably due to a slight misalinement of the pinhole collimator relative to the camera track or other parts of the apparatus. The error amounts to about 0.1 $\mathrm{mm}$, and would occur if the angle between the collimated beam and the camera track were as large as 5 minutes. 


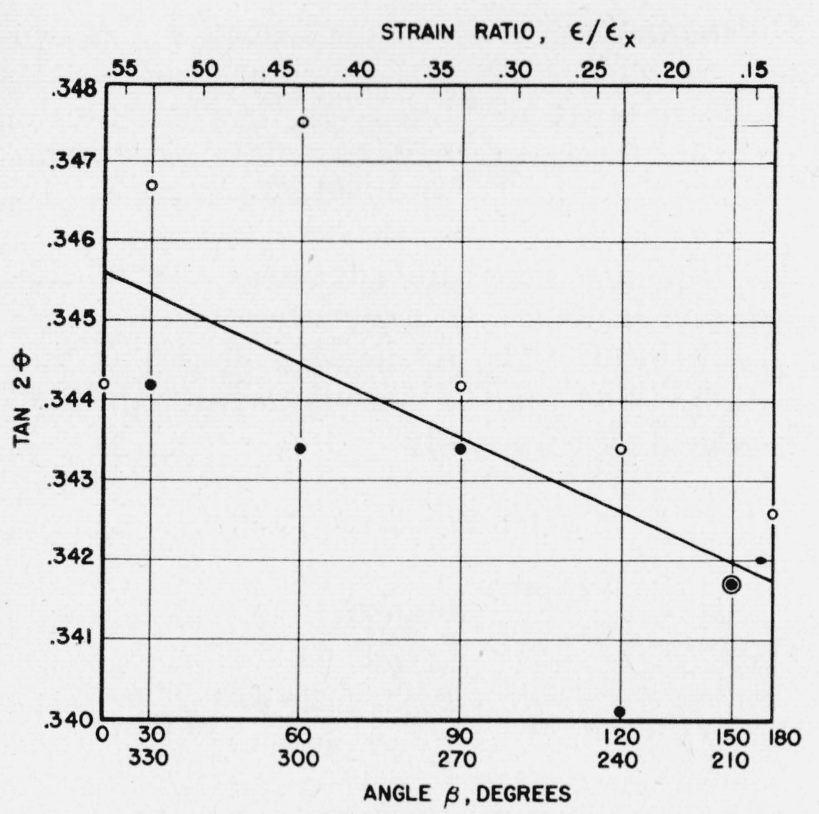

Figure 7. Typical plot of the measurements on one diffraction pattern.

The open circles represent values of 6 from 0 to $180^{\circ}$, closed circles from 210 to $330^{\circ}$. For convenience, the ordinates are drawn at the values of 6 where measurements were made, although the graph is actually constructed on a uniform strain ratio scale, as shown by the scale at the top.

\section{Results and Discussion}

The results obtained from a series of diffraction patterns from the flat specimen stressed by bending are shown in figure 8 . The values representing the $45^{\circ}$ incidence patterns are the intercepts for $\beta=0$, obtained as explained above, (fig. 7). The values for normal incidence are the averages of twelve readings from each diffraction pattern.

During the first stressing after normalizing, the specimen was inadvertently deformed enough to cause plastic deformation; consequently the surface layer of the specimen contained some residual compressive stress, as shown by the fact that the curves of figure 8 do not start together, but cross after the surface has been strained somewhat in tension.

It is interesting to note that the curve for $45^{\circ}$ incidence flattens off at a strain value of about $9 \times 10^{-4}$, indicating that plastic deformation occurred when the strain was greater than this value. However, the $90^{\circ}$ curve shows no flattening up to the maximum applied strain. From this it appears that plastic deformation in one direction in a crystal does not affect the atomic spacing or elastic properties in other directions where the strain is not great enough to cause slip.
In order to minimize systematic errors and utilize all of the data to obtain a single curve, the difference between values of $\tan 2 \theta$ for the two angles of incidence are plotted against strain in figure 9. (The subscripts A and B indicate values obtained with $90^{\circ}$ and $45^{\circ}$ incidence, respectively). Below the start of plastic deformation, the maximum deviation of any point from a linear relationship is 0.0035 -percent strain. This deviation corresponds to a stress of about $1,000 \mathrm{lb} / \mathrm{in}^{2}$. The dashed line on the figure is the theoretical relationship obtained from eq 1 and 2 on the assumption that Poisson's ratio $=0.3$, and that the stress is purely axial.

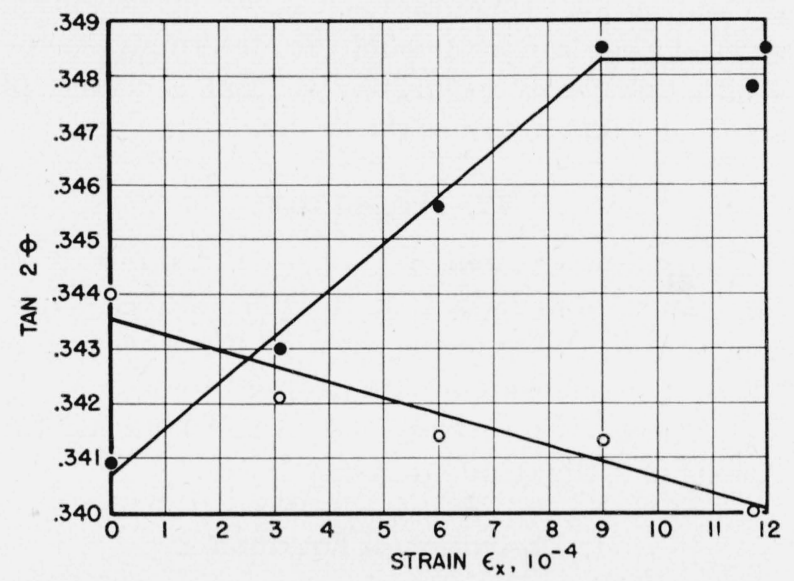

Figure 8. Effect of strain on the diffraction angle as measured with the X-ray beam incident at 90 and $45^{\circ}$.

Strain was measured with wire strain gages. Each of the points represents the data derived from one diffraction pattern. $\bigcirc, 90^{\circ}$ incidence; $\bullet 45^{\circ}$ incidence.

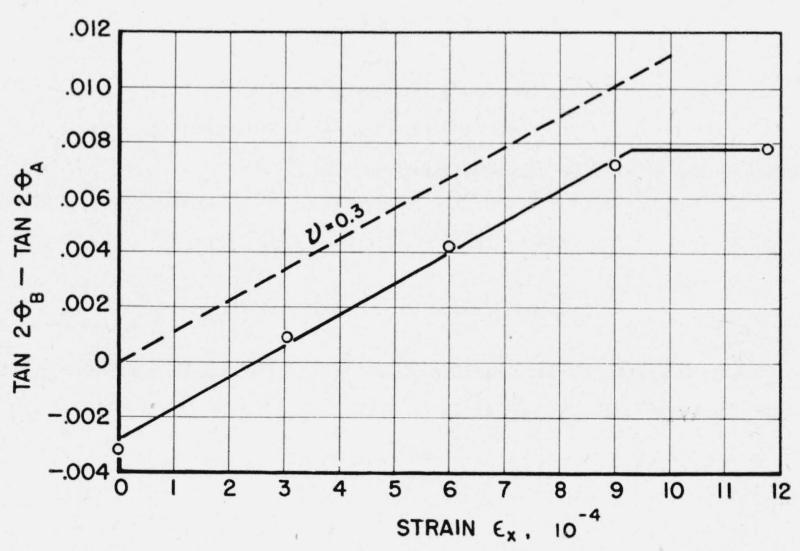

Figure 9. Difference between the 90 and $45^{\circ}$ points shown in figure 8.

The subscripts $A$ and $B$ indicate values of $\tan 2 \theta$ obtained with 90 and $45^{\circ}$ incidence, respectively. The dashed line is the theoretical curve calculated for Poisson's ratio $=0.3$. 
The slope of this theoretical curve is not changed greatly by change in the assumed value of Poisson's ratio, so that it is not possible to draw any conclusions regarding the correctness of the value assumed. However, the fact that the slope of the experimental curve is nearly the same as that of the theoretical curve shows that X-ray measurements can be used to give a reliable determination of strain in the direction of the applied stress.

\section{Conclusions}

For the condition of uniaxial stress, the precision of X-ray strain measurements can be improved by increasing the number of measurements of diffraction ring radius. This investigation showed that is was possible to determine elastic strain sufficiently accurately so that a change of stress of $1,000 \mathrm{lb} / \mathrm{in} .^{2}$ could be detected.

\section{Appendix}

The following symbols, not used in the body of the paper, will appear in the derivations below.

$a_{1}, a_{2}, a_{3}$, Direction cosines of the direction in which $\epsilon$ is measured with respect to the $X, Y$, and $Z$ axes, respectively. $\psi$, Angle between the $X$ axis and the incident $\mathrm{X}$-ray beam.

\section{Derivation of Equation 2}

Under the loading conditions described in section II, a, the directions of the principal strains coincide with the coordinate axes. The equation for the ellipsoid of strain can therefore be written

$$
\epsilon=a_{1}^{2} \epsilon_{X}+a_{2}^{2} \epsilon_{Y}+a_{3}{ }^{2} \epsilon_{Z} .
$$

As the stress is uniaxial, $\epsilon_{Y}=\epsilon_{Z}=-\nu \epsilon_{X}$. Also $a_{1}{ }^{2}+a_{2}{ }^{2}+a_{3}{ }^{2}=1$, so that eq. 4 becomes $\epsilon=\epsilon_{X}\left[a_{1}{ }^{2}-\right.$ $\left.\nu\left(1-a_{1}^{2}\right)\right]$. Or, assuming $\nu=0.3$

$$
\epsilon / \epsilon_{X}=\cos ^{2} \rho-0.3 \sin ^{2} \rho
$$

\section{Derivation of Equation 3}

To avoid confusion, the interplanar spacing in eq. 1 will be symbolized by $x$ rather than $d$ :

$$
n \lambda=2 x \sin \theta .
$$

\section{Differentiating}

$$
\begin{gathered}
x \cos \theta d \theta=-\sin \theta d x, \\
\frac{d x}{x}=\epsilon=-\cot \theta d \theta .
\end{gathered}
$$

This equation shows the advantage of the backreflection method (i. e., large value of $\theta$ ), for stress measurement. The relationship desired is that between $\epsilon$ and $d \tan 2 \theta$, since the latter is the term measured experimentally

$$
\begin{gathered}
d(\tan 2 \theta)=2 \sec ^{2} 2 \theta d \theta, \\
d \theta=\frac{\left(1-2 \sin ^{2} \theta\right)^{2}}{2} d \tan 2 \theta, \\
\epsilon=-\cot \theta d \theta=-\left[\frac{\left(1-2 \sin ^{2} \theta\right)^{2}}{2 \tan \theta}\right] d \tan 2 \theta .
\end{gathered}
$$

\section{Formulas for $\epsilon / \epsilon_{X}$}

Figure 10 shows the geometry for obtaining strain ratio as a function of $\beta$. The sides of the spherical triangle $X A C$ are related as

$$
\begin{aligned}
& \cos \rho=\cos (90-\theta) \cos \psi+ \\
& \sin (90-\theta) \sin \psi \cos \beta .
\end{aligned}
$$

For $\psi=45^{\circ}$ and $\theta=80 \frac{12^{\circ}}{}$ :

$$
\cos \rho=0.697+0.117 \cos \beta .
$$

For $\psi=90^{\circ}$ :

$$
\cos \rho=0.165 \cos \beta \text {. }
$$

Equation 2 can be written:

$$
\boldsymbol{\epsilon} / \boldsymbol{\epsilon}_{X}=1.3 \cos ^{2} \rho-0.3 \text {. }
$$

Substituting eq 7 and eq 8 , respectively,

$$
\begin{gathered}
\boldsymbol{\epsilon} / \epsilon_{X}=0.332+0.212 \cos \beta+0.018 \cos ^{2} \beta . \\
\boldsymbol{\epsilon} / \boldsymbol{\epsilon}_{X}=0.035 \cos ^{2} \beta-0.3
\end{gathered}
$$

These are the equations of the curves shown in figure 3 . 


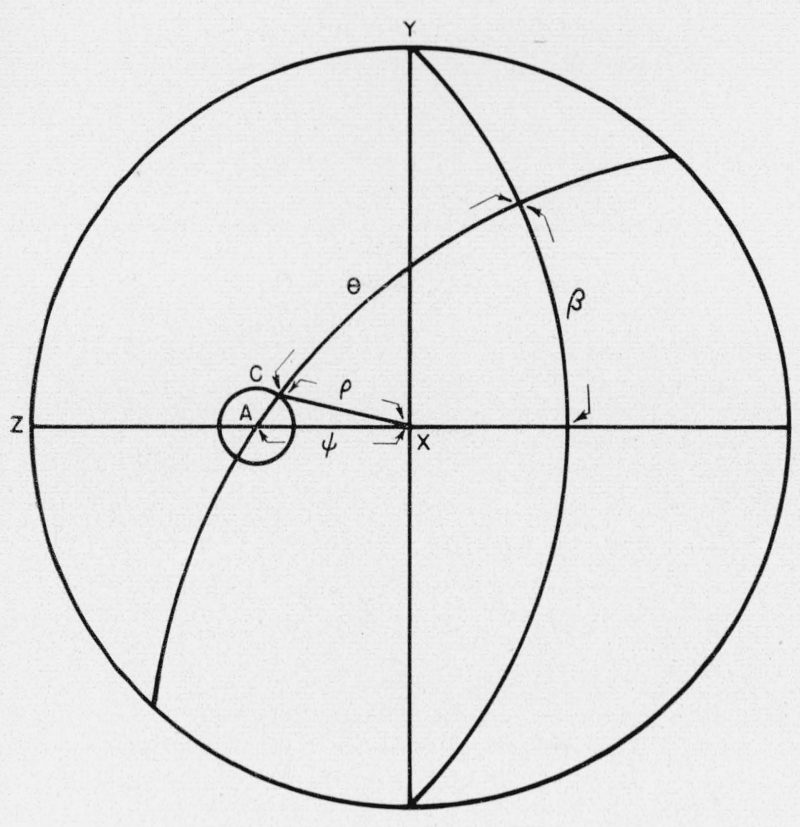

Figure 10. Stereographic projection in the $Y Z$ plane showing the geometrical considerations involved in the determination of strain ratio as a function of $b$. (See section VI, 3),

\section{Evaluation of Errors of Approximation}

The assumptions made in the following evalua-

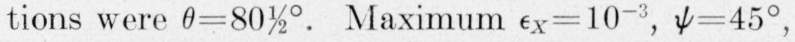
$\beta=0$; therefore $\epsilon / \epsilon_{X}=0.56 \epsilon=0.00056$.
In the following, a prime (') indicates that the value refers to the strained metal. From eq 1

$$
\begin{gathered}
d \sin \theta=d^{\prime} \sin \theta^{\prime} \\
\frac{d^{\prime}}{d}=\frac{\sin \theta}{\sin \theta^{\prime}}=1+\epsilon=1.00056 \\
\theta^{\prime}=80^{\circ} 19^{\prime}
\end{gathered}
$$

(a) Variation of the Term in Brackets in Equation 3 with Strain

$$
\begin{aligned}
& \frac{\left(1-2 \sin ^{2} \theta^{\prime}\right)^{2}}{2 \tan \theta^{\prime}}=0.0748 \\
& \frac{\left(1-2 \sin ^{2} \theta\right)^{2}}{2 \tan \theta}=0.0760
\end{aligned}
$$

That is, the change in $\tan 2 \theta$ is a linear function of strain within 1 percent over this range.

(b) Effect of Strain on Strain Ratio

From eq 6 and 2 the strain ratios for the above conditions are

$$
\begin{gathered}
\epsilon / \epsilon_{X}=0.562 \\
\epsilon^{\prime}{ }_{X}=0.566
\end{gathered}
$$

This change of less than 1 percent has a negligible effect on the determination of the intercept for $\beta=0$.

Washington, October 3, 1947. 